Originalien

Z Rheumatol 2020 $79: 135-142$

https://doi.org/10.1007/s00393-019-0613-z

Online publiziert: 12. März 2019

(c) Der/die Autor(en) 2019

\section{Redaktion}

U. Müller-Ladner, Bad Nauheim

U. Lange, Bad Nauheim

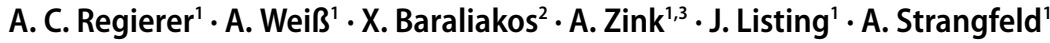 \\ 'Programmbereich Epidemiologie, Deutsches Rheuma-Forschungszentrum Berlin, Berlin, Deutschland \\ ${ }^{2}$ Rheumazentrum Ruhrgebiet Herne, Ruhr-Universität Bochum, Bochum, Deutschland \\ ${ }^{3}$ Department of Rheumatology and Clinical Immunology, Charité - Universitätsmedizin Berlin, Berlin, \\ Deutschland
}

\title{
RABBIT-SpA: ein neues Krankheitsregister für axiale Spondyloarthritis und Psoriasisarthritis
}

[2, 16]. Die Relevanz dieser Analysen für die Routineversorgung liegt v. a. in der Methodik des Registers als engmaschige Langzeitbeobachtung nicht selektierter Patienten des rheumatologischen Alltags begründet, die es erlaubt, besonders auch für solche Patienten, die in klinische Studien nicht eingeschlossen werden würden, Aussagen zu treffen.

Da sich das Studiendesign von RABBIT seit seiner Initiierung im Jahr 2001 sehr bewährt hat, wurden möglichst viele Aspekte und Prinzipien auch für das neue Register RABBIT-SpA übernommen. Ein wesentlicher Aspekt ist die gleichzeitige Beobachtung einer nicht exponierten Vergleichsgruppe („Kontrollgruppe") innerhalb des gleichen Studiendesigns. Bei RABBIT werden Patienten nach Versagen des ersten csDMARDs mit dem Beginn einer zweiten csDMARD-Therapie als Kontrollgruppe eingeschlossen. Aufgrund der unterschiedlichen Therapiealgorithmen bei axSpA und PsA gegenüber der RA mussten die Einschlusskriterien für die Kontrollgruppe bei RABBIT-SpA anders gewählt werden: Patienten mit einer axSpA können nach Versagen einer ersten Therapie (einschließlich des ersten NSAR) in die Kontrollgruppe eingeschlossen werden, wenn eine neue konventionelle Therapie begonnen wird. Hierzu zählt eine neue NSAR-Therapie, aber auch eine klinisch signifikante Dosiserhöhung eines NSAR ebenso wie

eine csDMARD-Therapie. Es steht $\mathrm{zu}$ erwarten, dass in die Kontrollgruppe eher Patienten mit niedrigerem Risiko eingeschlossen werden. Es ist aber auch möglich, dass einige der Patienten nicht lange in der Kontrollgruppe bleiben, weil sie eine rasche Therapieeskalation $\mathrm{zu}$ einem bDMARD benötigen.

Da für die PsA mit ihren unterschiedlichen Befallstypen verschiedene Therapiealgorithmen etabliert sind (s. GRAPPA-Guidelines [5]), wurde auch hier definiert, dass Patienten nach dem ersten Therapieversagen einer konventionellen Therapie in die Kontrollgruppe eingeschlossen werden können. Bei Patienten mit einem polyarthritischen Befallstyp ist also ein Einschluss z.B. nach Versagen von MTX und zu Beginn eines zweiten csDMARDs möglich. Patienten mit axialem Befall, für die der Algorithmus vorsieht, dass als Erstlinientherapie ein NSAR eingesetzt wird und als Zweitlinientherapie entweder ein anderes NSAR oder bereits eine bDMARD-Therapie begonnen wird, können auch hier zu Beginn des zweiten NSAR oder nach einer klinisch relevanten Dosiserhöhung eines NSAR in die Kontrollgruppe eingeschlossen werden.

Ein weiterer wesentlicher Aspekt des Designs von RABBIT sind die festgelegten Visitenzeiten. Diese orientieren sich an den üblichen Wiedervorstellungsrhythmen (zu Beginn nach 3, dann alle 6 Monate). Die festen Zeitintervalle zwischen 2 Messzeitpunkten dienen u. a. der Vollständigkeit und Vergleichbarkeit der samkeit oder zu Therapiekombinationen
gehören zu den Aufgaben des Registers 
Erfassung von unerwünschten Ereignissen. Bei RABBIT-SpA sind die gleichen Dokumentationszeitpunkte vorgesehen wie bei RABBIT-RA. Nach 5 Jahren sollen die Patienten erneut aufgeklärt und um die Einwilligung zur Dokumentation für weitere 5 Jahre gebeten werden. Dieses lange Follow-up von idealerweise 10 Jahren pro Patient trägt wesentlich zur Aussagefähigkeit der Langzeituntersuchungen bei. Hierdurch ist es möglich, umfassende Erkenntnisse über Therapiewechsel im Laufe der Erkrankung, bevorzugte Alterationen bzw. Faktoren, die die Reihenfolge der Therapien beeinflussen, und sekundäre Wirkversagen zu erhalten.

Bei RABBIT-SpA werden wie in RABBIT Angaben sowohl von den behandelnden Ärzten als auch von den Patienten erhoben. Die Diagnosestellung basiert auf der Arzteinschätzung, Klassifikationskriterien der Erkrankungen (ASAS- bzw. CASPAR-Kriterien) werden auf dem Basisbogen erhoben. Der Gelenkstatus wird bei der axSpA mit 44 Gelenken erhoben, bei der PsA mit 66 geschwollenen bzw. 68 druckschmerzhaften Gelenken. Die Enthesitis wird mit einem „Enthesitis-Männchen“ erfasst, aus dem der Spondyloarthritis Research Consortium of Canada(SPARCC)Enthesitis-Index berechnet werden kann. Neben den extraartikulären Manifestationen werden weitere Komorbiditäten erfasst. Bei Einschluss soll die Therapiehistorie angegeben und $\mathrm{zu}$ jedem Messzeitpunkt die jeweils aktuelle Therapie detailliert erfasst werden. Somit kann über den gesamten Beobachtungszeitraum der Therapieverlauf genau abgebildet werden. In den Folgebögen wechseln längere und kürzere Bögen ab. Unerwünschte Arzneimittelwirkungen werden ungeachtet eines möglichen Kausalzusammenhangs zur jeweiligen Therapie zu jedem Messzeitpunkt obligatorisch erfasst.

Bei der Entwicklung der Fragebögen wurde darauf geachtet, dass einerseits die für wissenschaftliche Analysen wesentlichen Parameter erhoben werden, andererseits die Belastung für die dokumentierenden Einrichtungen möglichst gering gehalten wird. So wurde z.B. auf die Erhebung des Bath Ankylosing
Spondylitis Metrology Index (BASMI) verzichtet. Es werden lediglich die lumbale Lateralflexion und das modifizierte Schober-Maß als Parameter der Wirbelsäulenbeweglichkeit erfragt. Bei der Einschätzung der Hautbeteiligung wurde auf den bei Dermatologen üblichen Psoriasis Area Severity Index (PASI) verzichtet, da dieser bei Rheumatologen nicht regelhaft durchgeführt wird. Lediglich der prozentuale Anteil der erkrankten Körperoberfläche wird erhoben.

Bei den Patientenfragebögen werden etablierte Instrumente eingesetzt, um die verschiedenen Aspekte der komplexen Krankheiten zu erfassen. Neben den bekannten krankheitsspezifischen Instrumenten zur Messung von Krankheitsaktivität, Funktion und Lebensqualität wie dem BASDAI, BASFI, ASAS-HI, HAQ, PsAID und DLQI enthalten die Patientenbögen Fragen zu beruflicher Situation und zur Arbeitsfähigkeit.

Der Hauptunterschied zwischen RABBIT und RABBIT-SpA liegt darin, dass für RABBIT-SpA ein Webbasiertes Online-Dokumentationssystem entwickelt wurde und das Projekt quasi papierlos arbeitet. Durch ein aufwendiges, mehrstufiges Pseudonymisierungsverfahren wird der Datenschutz gewährleistet. Das Dokumentationssystem wurde so entwickelt, dass trotz Pseudonymisierung eine Kommunikation der Studienleitung mit den Einrichtungen über einzelne Patienten möglich ist, um ein sorgfältiges Monitoring $\mathrm{zu}$ gewährleisten.

Ein Vorteil der elektronischen Dokumentation liegt unter anderem darin, dass durch Plausibilitätsprüfungen, die während der Eingabe der Daten erfolgen, direkt Fehler vermieden werden können, was zu weniger Rückfragen der Studienzentrale an die Einrichtungen führt. So können z. B. fehlerhafte Eintragungen von Datumsangaben, Messwerten etc. erkannt und von der dokumentierenden Person direkt verbessert werden. Die Daten stehen sofort für die Weiterverarbeitung zur Verfügung. Die Prüfung und Weiterverarbeitung ist in standardisierte Prozesse integriert, was die Fehleranfälligkeit verringert.

Die Patienten können ihre Fragebögen selbstständig online ausfüllen. Es gibt aber auch die Möglichkeit, dass in den Einrichtungen die Fragebögen für die Patienten ausgedruckt, vom Patienten im Wartezimmer ausgefüllt und anschließend vom Praxispersonal eingegeben werden. Wir erhoffen uns durch diese komfortable und flexible Lösung eine hohe Vollständigkeit der Patienten-berichteten Ergebnisparameter, da diese für die Einschätzung des Therapieverlaufs in der Rheumatologie eine herausragende Rolle spielen.

\section{Methodik}

RABBIT-SpA ist ein Krankheitsregister mit dem Studiendesign einer longitudinalen epidemiologischen Beobachtungsstudie. Durch Einschluss in das Register wird kein Einfluss auf die therapeutischen Entscheidungen der Ärzte genommen. Eingeschlossen werden können erwachsene Patienten mit einer axSpA oder einer PsA zu Beginn einer neuen Therapie, entweder in die Vergleichsgruppe („Kontrollgruppe") oder in die Index-Substanz-Gruppe (aktuell [Stand: Dezember 2018] sind dies für axSpA: Adalimumab [Humira ${ }^{\circledR}$, AbbVie Deutschland GmbH \& Co. KG, Wiesbaden, Deutschland], Certolizumab [Cimzia ${ }^{\circledR}$, UCB Pharma GmbH, Monheim, Deutschland], Etanercept [Enbrel ${ }^{\circledR}$, Pfizer Pharma $\mathrm{GmbH}$, Berlin, Deutschland], Golimumab [Simponi ${ }^{\circledR}$, MSD Sharp \& Dohme $\mathrm{GmbH}$, Haar, Deutschland] und Secukinumab [Cosentyx ${ }^{\circledR}$, Novartis Pharma GmbH, Nürnberg, Deutschland]; für die PsA: Adalimumab [Humira ${ }^{\circledR}$, AbbVie Deutschland GmbH \& Co. KG, Wiesbaden, Deutschland], Apremilast [Otezla ${ }^{\circledR}$, Celgene International II Sarl, Couvet, Schweiz], Certolizumab [Cimzia ${ }^{\circledR}, \mathrm{UCB}$ Pharma GmbH, Monheim, Deutschland], Etanercept [Enbrel ${ }^{\circledR}$, Pfizer Pharma GmbH, Berlin, Deutschland], Golimumab [Simponi ${ }^{\circledR}$, MSD Sharp \& Dohme GmbH, Haar, Deutschland], Ixekizumab [Taltz ${ }^{\circledR}$, Lilly Deutschland $\mathrm{GmbH}$, Bad Homburg, Deutschland], Secukinumab [Cosentyx ${ }^{\circledR}$, Novartis Pharma GmbH, Nürnberg, Deutschland], Tofacitinib [Xeljanz ${ }^{\circledR}$,Pfizer Pharma GmbH, Berlin, Deutschland] und Ustekinumab [Stelara $^{\circledR}$, Janssen-Cilag GmbH, Neuss, Deutschland]). Für axSpA-Patienten ist 
Z Rheumatol 2020 · 79:135-142 https://doi.org/10.1007/s00393-019-0613-Z

(c) Der/die Autor(en) 2019

\section{A. C. Regierer · A. Weiß X X. Baraliakos · A. Zink · J. Listing · A. Strangfeld}

\section{RABBIT-SpA: ein neues Krankheitsregister für axiale Spondyloarthritis und Psoriasisarthritis}

\section{Zusammenfassung}

Hintergrund. Durch die Zulassung einer Reihe neuer Biologika mit verschiedenen Wirkmechanismen sowie den zunehmenden Einsatz von Biosimilars hat sich die Behandlung der axialen Spondyloarthritis (axSpA) und der Psoriasisarthritis (PsA) in den letzten Jahren enorm gewandelt. Die Datenlage hinsichtlich der langfristigen Sicherheit und Wirksamkeit unter Alltagsbedingungen ist bisher nicht ausreichend. Deshalb wurde im Deutschen Rheuma-Forschungszentrum eine neue Kohortenstudie begonnen.

Ziel der Arbeit. Vorstellung erster Ergebnisse aus dem im Mai 2017 gestarteten Register RABBIT-SpA.

Material und Methoden. Prospektive longitudinale Kohortenstudie, angelehnt an das RABBIT-Design: Einschluss bei Beginn oder Wechsel einer Therapie entweder in die Vergleichsgruppe (konventionelle systemische Therapie, einschließlich NSAR) oder in die Gruppe der sog. Indexmedikamente. Die Beobachtungszeit sollte pro Patient mindestens 5 und möglichst 10 Jahre betragen. RABBIT-SpA benutzt ein Internetgestütztes Dokumentationssystem. Ergebnisse. Bis Mitte Dezember 2018 wurden 514 axSpA-Patienten in RABBIT-SpA dokumentiert, 410 mit einem Indexmedikament und 104 mit einer konventionellen Therapie. Zwischen diesen Behandlungsgruppen bestehen Unterschiede z. B. in der Krankheitsdauer und in Parametern der Krankheitsaktivität. Weiterhin fällt auf, dass bei den axSpA-Patienten ca. 5 Jahre zwischen dem Symptombeginn und der Diagnosesicherung liegen.

Von den 355 PsA-Patienten wurden 265 mit einem Indexmedikament und 90 mit einer konventionellen Therapie eingeschlossen.
Bei $86 \%$ der PsA-Patienten besteht ein dominant peripherer Befall. Die Anzahl der druckschmerzhaften Gelenke liegt im Mittel bei 8 und die Anzahl der geschwollenen Gelenke im Mittel bei 4 .

Diskussion. Das online-Register RABBIT-SpA wird von den beteiligten rheumatologischen Einrichtungen gut angenommen. Die elektronische Erfassung der Patientendaten kann in einer angemessenen Zeit und mit einem vertretbaren Aufwand erfolgen. Die Teilnahme weiterer rheumatologischer Einrichtungen an RABBIT-SpA ist jederzeit möglich.

\section{Schlüsselwörter}

Axiale Spondyloarthritis · Psoriasisarthritis . DMARDs · Langzeitsicherheit - Wirksamkeit . Pharmakoepidemiologie

\section{RABBIT-SpA: a new disease register for axial spondyloarthritis and psoriatic arthritis}

\section{Abstract}

Background. The treatment of axial spondyloarthritis (axSpA) and psoriatic arthritis (PsA) has changed enormously in recent years due to market authorization of a number of new biologicals with different modes of action and the increasing use of biosimilars. Real-world data on long-term safety and efficacy under routine daily conditions is not yet sufficient. Therefore, the German Rheumatism Research Center has initiated a new cohort study covering axSpA and PsA.

Objective. Presentation of initial results from the new register RABBIT-SpA, which was started in May 2017.

Material and methods. This is a prospective longitudinal cohort study with a similar study design to the German biologics register RABBIT. Patients can be included at the start of a new treatment either in the so-called index drug group or in the comparison group (conventional systemic treatment, including non-steroidal anti-inflammatory drugs, NSAID). Follow-up per patient should be at least 5 years and preferably 10 years. The RABBIT-SpA uses a web-based documentation system.

Results. Up to mid-December 2018 a total of 514 axSpA patients had been documented in RABBIT-SpA, 410 with an index drug and 104 with conventional treatment. There are differences between these treatment groups, e. g. in the duration of the disease and in parameters of disease activity. It is also noticeable that in axSpA patients, approximately 5 years lie between the onset of the symptoms and confirmation of the diagnosis. Of the 355 PsA patients, 265 were included with an index drug and 90 with conventional treatment. Of the PsA patients $86 \%$ have a dominant peripheral manifestation. The average number of pressure tender joints is 8 and the average number of swollen joints is 4 .

Conclusion. The online register RABBITSpA is well-received by the participating rheumatological institutions. The electronic recording of patient data can be carried out in a reasonable time. Participation in the RABBIT-SpA is open to new rheumatological institutions at any time.

Keywords

Axial spondyloarthritis - Psoriatic arthritis . DMARDs · Drug safety - Effectiveness .

Pharmacoepidemiology ein Einschluss in die Kontrollgruppe möglich bei Beginn eines neuen NSAR, bei einer klinisch relevanten Dosiserhöhung einer laufenden NSAR-Therapie oder bei Beginn einer Therapie mit einem konventionellen synthetischen DMARD. Bei PsA-Patienten ist ein Einschluss möglich bei Beginn einer systemischen Standardtherapie (nach Versagen mindestens einer vorherigen
Standardtherapie), wobei z. B. bei axialem Befall auch eine NSAR-Therapie als Standardtherapie gilt.

Im Mai 2017 wurde mit der Dokumentation der axSpA-Patienten begonnen, seit Oktober 2017 können auch Patienten mit PsA eingeschlossen werden. Nachfolgend werden erste Ergebnisse aus beiden Kohorten in Bezug auf ihre Baseline-Charakteristika mit einem Daten- stand vom 31.08.2018 dargestellt. Alle Analysen erfolgten mit dem Statistikprogramm SAS, Version 9.4. Eine Einwilligungserklärung wurde von allen Studienteilnehmern unterschrieben. Ein positives Ethikvotum wurde im August 2016 von der Ethikkommission der Charité Universitätsmedizin Berlin erteilt. 
Tab. 1 axSpA: Baseline-Charakteristika

\begin{tabular}{|c|c|c|}
\hline Parameter & Index & Kontrolle \\
\hline$N$ & 410 & 104 \\
\hline Frauen, $n(\%)$ & $169(41)$ & $53(51)$ \\
\hline Alter, MW (SD) & $43,5(12,5)$ & $43(13,5)$ \\
\hline BMI, MW (SD) & $26,5(5,1)$ & $26,1(4,5)$ \\
\hline Symptomdauer in Jahren, MW (SD) & $12,6(11,1)$ & $9,3(11)$ \\
\hline Dauer seit Diagnosestellung in Jahren, MW (SD) & $7,2(9,5)$ & $4,5(8,7)$ \\
\hline nr-axSpA, $n(\%)$ & $64(25,9)$ & $17(42,5)$ \\
\hline HLA-B27 positiv, $n(\%)$ & $302(74,2)$ & $63(63)$ \\
\hline Entzündlicher Rückenschmerz, aktuell, $n$ (\%) & $356(87,5)$ & $81(81)$ \\
\hline Uveitis, jemals, $n$ (\%) & $71(17,3)$ & $19(18,3)$ \\
\hline Psoriasis, jemals, $n$ (\%) & $60(14,6)$ & $11(10,6)$ \\
\hline CED, jemals, $n(\%)$ & $28(6,8)$ & $5(4,8)$ \\
\hline MW Mittelwert, SD Standardabweichung & & \\
\hline
\end{tabular}

Tab. 2 axSpA: Krankheitsaktivität

\begin{tabular}{|l|l|l|}
\hline Parameter & Index & Kontrolle \\
\hline Krankheitsaktivität gesamt (Arzteinschätzung) (0-10), MW (SD) & $5,9(1,7)$ & $4,8(1,9)$ \\
\hline CRP mg/l, MW (SD) & $12,5(18)$ & $12,8(19,9)$ \\
\hline CRP >5 mg/l, $n$ (\%) & $220(60,9)$ & $38(55,1)$ \\
\hline Arthritis, aktuell, $n$ (\%) & $95(23,4)$ & $29(29)$ \\
\hline $\begin{array}{l}\text { Arthritis, Anzahl der Gelenke (44), MW (SD) } \\
\text { Enthesitis, aktuell, } n \text { (\%) }\end{array}$ & $0,9(3)$ & $1(2)$ \\
\hline Enthesitis, Anzahl der betroffenen Sehnenansätze (16, SPARCC), & $65(16)$ & $19(19,2)$ \\
\hline MW (SD) & $0,4(1,3)$ & $0,7(1,7)$ \\
\hline BASDAl, MW (SD) & $4,7(1,9)$ & $4,5(1,9)$ \\
\hline ASDAS, MW (SD) & $3(0,9)$ & $2,8(1)$ \\
\hline BASFI (0-10), MW (SD) & $4(2,3)$ & $3,4(2,3)$ \\
\hline Schmerz (0-10), MW (SD) & $5,9(2,3)$ & $5,5(2,4)$ \\
\hline Krankheitsaktivität (Patienteneinschätzung) (0-10), MW (SD) & $6(2,3)$ & $5,7(2,5)$ \\
\hline Gesundheitszustand (Patienteneinschätzung) (0-10), MW (SD) & $5,4(2,1)$ & $5,1(2,2)$ \\
\hline Stärke der Morgensteifigkeit (0-10), MW (SD) & $4,9(2,7)$ & $5,1(2,9)$ \\
\hline MW Mittelwert, SD Standardabweichung & & \\
\hline
\end{tabular}

\section{Ergebnisse}

\section{Axiale Spondyloarthritis}

Bis Mitte Dezember 2018 wurden 514 Patienten mit axSpA in RABBIT-SpA dokumentiert. Von diesen wurden $410 \mathrm{mit}$ einem Biologikum oder Biosimilar und 104 mit einer konventionellen Therapie eingeschlossen. In $92 \%$ der Fälle war dies ein NSAR. Die Baseline-Charakteristika sind in Tab. 1 dargestellt. Patienten in der Kontrollgruppe sind etwas häufiger Frauen und haben eine kürzere Krankheitsdauer. Auffallend ist u. a., dass weiterhin eine Zeitspanne von ca. 5 Jahren besteht zwischen den ersten Symptomen und der Diagnosestellung.

In - Tab. 2 sind Krankheitsaktivitätsparameter der axSpA-Patienten dargestellt. Sowohl der Bath Ankylosing Spondylitis Disease Activity Index (BASDAI) als auch der Ankylosing Spondylitis Disease Activity Score (ASDAS) zeigen in beiden Therapiegruppen hohe Mittelwerte. Trotzdem sind Patienten der Indexgruppe in fast allen Parametern etwas stärker betroffen.

Passend zu dem eher jüngeren Alter der axSpA-Patienten haben $44 \%$ der Indexgruppe bzw. $53 \%$ der Kontrollgruppe keine weitere Erkrankung. Drei oder mehr Komorbiditäten sind bei Patien- ten der Indexgruppe deutlich häufiger (21\%) als bei Patienten der Kontrollgruppe $(14 \%)$. Insgesamt sind die häufigsten angegebenen Komorbiditäten arterielle Hypertonie (20\%), degenerative Wirbelsäulenerkrankungen (14\%) und Depression $(8 \%)$.

\section{Psoriasisarthritis}

Bis Mitte Dezember 2018 wurden insgesamt 355 Patienten mit einer PsA eingeschlossen (• Tab. 3). Der Frauenanteil und das mittlere Alter der PsA-Patienten liegen, wie zu erwarten war, höher als bei der axSpA. Auch der mittlere BMI ist mit 28,5 etwas höher als bei axSpA-Patienten mit 26,5. Das Zeitintervall zwischen Symptombeginn der Gelenkbeschwerden und Diagnosestellung der PsA ist mit 3 Jahren etwas kürzer als bei der axSpA, dem Gelenkbefall gehen jedoch häufig viele Jahre Hautbefall voraus. Die meisten Patienten zeigen einen dominant peripheren Befallstyp.

Parameter der Krankheitsaktivität der PsA-Patienten sind in $\bullet$ Tab. 4 dargestellt. Hier zeigt sich, dass Patienten in der Indexsubstanzgruppe bis auf den Parameter „Krankheitsaktivität Haut“ (Arzteinschätzung) im Mittel etwas stärker betroffen sind.

Wie aus der Literatur bekannt, leiden PsA-Patienten unter vielen weiteren Komorbiditäten. In der Indextherapiegruppe gaben $31 \%$ der Patienten keine weitere Erkrankung an, in der Kontrollgruppe $52 \%$ der Patienten. Auch der Anteil multimorbider Patienten mit 3 oder mehr Komorbiditäten war in der Indexgruppe deutlich häufiger als in der Kontrollgruppe (33\% vs. $12 \%)$. Insgesamt sind die häufigsten Komorbiditäten in der hier beschriebenen Kohorte arterielle Hypertonie (34\%), degenerative Wirbelsäulenerkrankungen (18\%) und Depression (11\%).

\section{Diskussion}

Die Etablierung einer neuen langfristigen Kohortenstudie am DRFZ hat eine Vielzahl von Gründen. Ein wesentlicher Grund besteht darin, dass die für die RA vorhandenen Daten bezüglich der Wirksamkeit und Sicherheit der Biolo- 
Hier steht eine Anzeige.

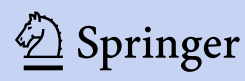


Tab. 3 PsA:Baseline-Charakteristika

\begin{tabular}{|c|c|c|c|}
\hline \multicolumn{2}{|c|}{ Parameter } & Index & Kontrolle \\
\hline \multicolumn{2}{|l|}{$N$} & 265 & 90 \\
\hline \multicolumn{2}{|c|}{ Frauen, $n(\%)$} & $147(55,5)$ & $57(63,3)$ \\
\hline \multicolumn{2}{|c|}{ Alter, MW (SD) } & $50,8(12,1)$ & $52,4(11,1)$ \\
\hline \multicolumn{2}{|c|}{ BMI, MW (SD) } & $28,4(5,4)$ & $29(5,8)$ \\
\hline \multicolumn{2}{|c|}{ Symptomdauer (Haut) in Jahren, MW (SD) } & $18(14,4)$ & $18,3(16,5)$ \\
\hline \multicolumn{2}{|c|}{ Dauer seit Diagnosestellung der Psoriasis in Jahren, MW (SD) } & $14,7(13,6)$ & $17,2(16,4)$ \\
\hline \multicolumn{2}{|c|}{ Symptomdauer (Gelenke) in Jahren, MW (SD) } & $10,4(9,2)$ & $7,8(9,9)$ \\
\hline \multicolumn{2}{|c|}{ Dauer seit Diagnosestellung der PsA in Jahren, MW (SD) } & $7,3(7,5)$ & $5,2(8,4)$ \\
\hline \multirow[t]{4}{*}{ Befallstyp } & Dominant axial, $n(\%)$ & $23(8,8)$ & $4(5,7)$ \\
\hline & DIP-Arthritis, $n$ (\%) & $14(5,3)$ & $2(2,9)$ \\
\hline & Arthritis mutilans, $n(\%)$ & $3(1,1)$ & $2(2,9)$ \\
\hline & Dominant peripher, $n(\%)$ & $222(84,7)$ & $62(88,6)$ \\
\hline \multicolumn{2}{|c|}{ Patienten mit druckschmerzhaften Gelenken, $n$ (\%) } & $219(82,6)$ & $59(83,1)$ \\
\hline \multicolumn{2}{|c|}{ Anzahl druckschmerzhafter Gelenke, MW (SD) } & $7,4(8,1)$ & $5,5(6,1)$ \\
\hline \multicolumn{2}{|c|}{ Patienten mit geschwollenen Gelenken, $n$ (\%) } & $163(61,7)$ & $50(70,4)$ \\
\hline \multicolumn{2}{|c|}{ Anzahl geschwollener Gelenke, MW (SD) } & $3(4,3)$ & $3,3(4,6)$ \\
\hline \multicolumn{2}{|c|}{ Enthesitis (aktuell), $n$ (\%) } & $49(27,1)$ & $10(17,9)$ \\
\hline \multicolumn{2}{|c|}{ Daktylitis (aktuell), $n$ (\%) } & $54(20,5)$ & $15(21,1)$ \\
\hline \multicolumn{2}{|c|}{ Axialer Befall (aktuell), $n$ (\%) } & $64(24,2)$ & $8(11,3)$ \\
\hline \multicolumn{2}{|c|}{ Nagelpsoriasis (aktuell), $n$ (\%) } & $118(44,9)$ & $34(47,2)$ \\
\hline \multicolumn{2}{|c|}{ Uveitis (jemals), $n$ (\%) } & $1(0,5)$ & $1(1,4)$ \\
\hline \multicolumn{2}{|c|}{ CED (jemals), $n(\%)$} & $8(3)$ & - \\
\hline \multicolumn{4}{|c|}{ MW Mittelwert, SD Standardabweichung } \\
\hline
\end{tabular}

Tab. 4 PsA: Krankheitsaktivität

\begin{tabular}{|l|l|l|}
\hline Parameter & Index & Kontrolle \\
\hline Krankheitsaktivität gesamt (Arzteinschätzung) (0-10), MW (SD) & $5,6(2)$ & $5(2)$ \\
\hline Krankheitsaktivität Haut (Arzteinschätzung) (0-10), MW (SD) & $3,3(2,7)$ & $3,7(2,7)$ \\
\hline Krankheitsaktivität Gelenke (Arzteinschätzung) (0-10), MW (SD) & $5,3(2,3)$ & $4,7(2,3)$ \\
\hline Befallene Körperoberfläche (BSA), MW (SD) & $8,7(14,8)$ & $9,6(15,6)$ \\
\hline Schmerz (0-10), MW (SD) & $5,4(2,4)$ & $5,4(2,4)$ \\
\hline Krankheitsaktivität (Patienteneinschätzung) (0-10), MW (SD) & $5,5(2,3)$ & $5,5(2,4)$ \\
\hline Gesundheitszustand (Patienteneinschätzung) (0-10), MW (SD) & $5,3(2,1)$ & $5,2(2,1)$ \\
\hline DLQI (0-30), MW (SD) & $5,5(6,3)$ & $5,2(6,5)$ \\
\hline MW Mittelwert, SD Standardabweichung & & \\
\hline
\end{tabular}

gika, Biosimilars und tsDMARDs nicht unhinterfragt auf die anderen zugelassenen Indikationen übertragen werden können [7]. AxSpA, PsA und RA sind Erkrankungen mit deutlichen Unterschieden in vielen Patientencharakteristika, wie z. B. der Geschlechtsverteilung, dem Alter und den Komorbiditäten. Zudem gibt es wesentliche Unterschiede in den Therapiealgorithmen, in dem Verlauf der Erkrankungen und den pathophysiologischen Hintergründen. Auch wenn ein gewisser Überlappungsbereich existiert, ist eine sorgfältige Analyse der verschiedenen Entitäten notwendig.

Ein weiterer wichtiger Grund für das neue Register besteht darin, dass die eingesetzten Medikamente aufgrund der zugrunde liegenden Pathophysiologien bei den verschiedenen Erkrankungen unterschiedlich gut wirksam sind. So sind z. B. die IL-17-Inhibitoren bei den Spondyloarthritiden sehr vielversprechend [1, 11], während sie bei der RA keine Rolle zu spielen scheinen [3]. IL-12/23-Inhibitoren sind wiederum bei der PsA sehr wirk- sam, bei der axSpA dagegen nur gering $[6,9]$. Die Pipeline an innovativen Medikamenten ist insbesondere bei der PsA, aber auch bei der axSpA beeindruckend. Für beide Entitäten befindet sich eine Reihe von Medikamenten in der Endphase der klinischen Erprobung. Im Sinne der Patientensicherheit ist es ausgesprochen wichtig, für alle neuen Medikamente unabhängige, gut monitorierte Beobachtungsdaten zu generieren, um die Sicherheit und Wirksamkeit in einer nicht selektierten Patientenpopulation und mit einer langen Beobachtungsdauer analysieren zu können.

Auch der zunehmende Einsatz von Biosimilars ist ein wichtiger Grund für den Aufbau des SpA-Registers. Aus unserer Sicht besteht hier eine Wissenslücke bezüglich der Langzeitwirksamkeit und -sicherheit, insbesondere deshalb, weil aufgrund der für die Biosimilars geltenden Zulassungsverfahren sehr viel weniger Daten aus klinischen Studien zur Verfügung stehen. So gilt hier z.B. das Prinzip der Extrapolation, dies bedeutet, dass die Hersteller nicht für alle $\mathrm{Zu}$ lassungsindikationen klinische Prüfungen durchführen müssen, sondern dass, wenn die Biosimilarität für eine Indikation gezeigt werden konnte, dies ohne weitere Studien auf die anderen Indikationen ausgeweitet werden kann [15].

Diese erste Auswertung der beiden Kohorten zeigt schlüssige Werte in Bezug auf die demografischen und klinischen Charakteristika der eingeschlossenen Patienten. Die beiden Krankheitskohorten unterscheiden sich erwartungsgemäß z. B. im Alter, dem Geschlechterverhältnis und dem BMI.

Die immer noch große Diagnoseverzögerung bei der axSpA von rund 5 Jahren unterstreicht die Notwendigkeit einer rascheren Diagnostik und Überweisung zum Rheumatologen.

Als Einschlusskriterium in das Register wird ein Therapiewechsel bzw. eine Intensivierung verlangt. Dazu passen die relativ hohen Mittelwerte der verschiedenen Krankheitsaktivitätsparameter zu Baseline, die die Notwendigkeit für einen Therapiewechsel verdeutlichen. Die Patienten der Indextherapiegruppe sind in fast allen Parametern etwas stärker betroffen als Patienten der Kontrollgruppe. 
Dies erscheint plausibel angesichts der Therapieentscheidung Intensivierung vs. Wechsel, die der Zuordnung zu den beiden Gruppen zugrunde liegt. Wir erwarten schwerere Krankheitsverläufe bei den Patienten, die mit Biologika oder anderen Indexmedikamenten behandelt werden.

Eine wesentliche Schwierigkeit bei Beobachtungsstudien mit ihrer fehlenden Randomisierung liegt darin, dass Ärzte selbstverständlich bewusst Therapieentscheidungen für den individuellen Patienten vornehmen und hierbei bekannte Faktoren, wie z. B. Komorbiditäten, aber auch Patientenpräferenz und den eigenen Erfahrungsschatz für die Therapieentscheidung zugrunde legen. Dies bedeutet, dass es bei Beobachtungsstudien immer einen sog. Indikationsbias gibt. Die Patienten, für die Therapie $\mathrm{X}$ ausgewählt wurde, unterscheiden sich hinsichtlich verschiedener Einflussfaktoren von Patienten, für die Therapie Y ausgewählt wurde. In RABBIT haben die Patienten, die unter konventionellen DMARDs eingeschlossen werden (Kontrollgruppe) z.B. eine kürzere Krankheitsdauer und eine etwas niedrigere Krankheitsaktivität als Patienten, die mit Beginn einer Biologikatherapie erfasst werden. Diese Unterschiede müssen bei Vergleichen zwischen den Therapiegruppen berücksichtigt werden, d.h. die Gruppen müssen mittels statistischer Methoden (Regressionsansätze oder Propensity Score-basierte Methoden) vergleichbar gemacht werden, um auf dieser Basis Unterschiede zwischen den Gruppen zu untersuchen und statistisch bewerten zu können.

Die Bedeutung von Daten aus sorgfältig monitorierten Beobachtungsstudien wird zunehmend wahrgenommen. Aus unserer Sicht sind sie insbesondere für die Bewertung der Langzeitsicherheit von medikamentösen Therapien unersetzlich. Dies zeigt sich auch durch eine zunehmende Berücksichtigung von Beobachtungsdaten durch die Regulationsbehörden. Die EMA unternimmt verschiedene Ansätze, um Evidenz aus Beobachtungsstudien und anderen „Real-World-Daten“ besser verwenden $\mathrm{zu}$ können, so gibt es z.B. eine Initiative, Patientenregister mit bestimmten Qualitätsstandards zu fördern. RABBIT fungiert hierbei als eines der Vorbildregister.

Wir freuen uns, wenn weitere Einrichtungen bei RABBIT-SpA mitmachen und möglichst viele ihrer Patienten in das Register einschließen. Nur durch die aktive Mitarbeit einer großen Zahl von Rheumatologinnen und Rheumatologen wird es möglich sein, aufgrund einer soliden, die Behandlungsrealität abbildenden Datenbasis, klinisch relevante Fragestellungen beantworten zu können.

\section{Korrespondenzadresse}

\section{PD Dr. A.C. Regierer}

Programmbereich Epidemiologie, Deutsches Rheuma-Forschungszentrum Berlin Charitéplatz 1, 10117 Berlin, Deutschland anne.regierer@drfz.de

Danksagung. Wir bedanken uns bei allen Patienten, die eingewilligt haben, bei RABBIT-SpA teilzunehmen. Unser Dank geht weiterhin an alle teilnehmenden Ärzte und Mitarbeiter in den beteiligten Einrichtungen.

Förderung. RABBIT-SpA wird gemeinschaftlich finanziert durch AbbVie, Celgene, Janssen-Cilag, Lilly, MSD, Novartis, Pfizer und UCB. Die Studienleitung am DRFZ ist unabhängig in der Durchführung der Studie, den Auswertungen und der Publikation der Ergebnisse.

\section{Einhaltung ethischer Richtlinien}

Interessenkonflikt. A.C. Regierer, A. Weiß, X. Baraliakos, A. Zink, J. Listing und A. Strangfeld geben an, dass kein Interessenkonflikt besteht.

Alle im vorliegenden Manuskript beschriebenen Untersuchungen am Menschen wurden mit Zustimmung der zuständigen Ethik-Kommission, im Einklang mit nationalem Recht sowie gemäß der Deklaration von Helsinki von 1975 in der aktuellen, überarbeiteten Fassung durchgeführt. Von allen beteiligten Patienten liegt eine Einverständniserklärung vor.

Open Access. Dieser Artikel wird unter der Creative Commons Namensnennung 4.0 International Lizenz (http://creativecommons.org/licenses/by/4.0/deed. de) veröffentlicht, welche die Nutzung, Vervielfältigung, Bearbeitung, Verbreitung und Wiedergabe in jeglichem Medium und Format erlaubt, sofern Sie den/die ursprünglichen Autor(en) und die Quelle ordnungsgemäßnennen, einen Linkzur Creative Commons Lizenz beifügen und angeben, ob Änderungen vorgenommen wurden.

\section{Literatur}

1. Baeten D, Sieper J, Braun J et al (2015) Secukinumab, an Interleukin-17A inhibitor, in Ankylosing Spondylitis. NEngl J Med 373:2534-2548

2. Baganz L, Richter A, Kekow J et al (2018) Longterm effectiveness of tocilizumab in patients with rheumatoid arthritis, stratified by number of previous treatment failures with biologic agents: results from the German RABBIT cohort. Rheumatol Int 38:579-587

3. Blanco FJ, Moricke R, Dokoupilova E et al (2017) Secukinumab in active rheumatoid arthritis: a phase III randomized, double-blind, active Comparator- and placebo-controlled study. Arthritis Rheumatol 69:1144-1153

4. Braun J (2016) New targets in psoriatic arthritis. Rheumatology (Oxf) 55:ii30-ii37

5. Coates LC, Kavanaugh A, Mease PJ et al (2016) Group for research and assessment of psoriasis and Psoriatic arthritis 2015 treatment recommendations for Psoriatic arthritis. Arthritis Rheumatol 68:1060-1071

6. Deodhar A, Gensler LS, Sieper J et al (2018) Three multicenter, randomized, double-blind, placebocontrolled studies evaluating the efficacy and safety of Ustekinumab in axial Spondyloarthritis. Arthritis Rheumatol. https://doi.org/10.1002/art. 40728

7. Garcia-Doval I, Hernandez MV, Vanaclocha F et al (2017) Should tumour necrosis factor antagonist safety information be applied from patients with rheumatoid arthritis to psoriasis? Rates of serious adverse events in the prospective rheumatoid arthritisBIOBADASERand psoriasis BIOBADADERM cohorts. Br J Dermatol 176:643-649

8. Gossec L, Smolen JS, Ramiro S et al (2016) European League Against Rheumatism (EULAR) recommendations for the management of psoriatic arthritis with pharmacological therapies: 2015 update. Ann Rheum Dis 75:499-510

9. Kavanaugh A, Ritchlin C, Rahman P et al (2014) Ustekinumab, an anti-IL-12/23 p40 monoclonal antibody, inhibits radiographic progression in patients with active psoriatic arthritis: results of an integrated analysis of radiographic data from the phase 3, multicentre, randomised, double-blind, placebo-controlled PSUMMIT-1 and PSUMMIT-2 trials. Ann Rheum Dis 73:1000-1006

10. Listing J, Kekow J, Manger B et al (2015) Mortality in rheumatoid arthritis: the impact of disease activity, treatment with glucocorticoids, TNFalpha inhibitors and rituximab. Ann Rheum Dis 74:415-421

11. Mease PJ, Mcinnes IB, Kirkham B et al (2015) Secukinumab inhibition of Interleukin-17A in patients with Psoriatic arthritis. N Engl J Med 373:1329-1339

12. Meissner Y, Zink A, Kekow J et al (2016) Impact of disease activity and treatment of comorbidities on the risk of myocardial infarction in rheumatoid arthritis. Arthritis Res Ther 18:183

13. Mercer LK, Askling J, Raaschou P et al (2017) Risk of invasive melanoma in patients with rheumatoid arthritis treated with biologics: results from a collaborative project of 11 European biologic registers. Ann Rheum Dis 76:386-391

14. Mercer LK, Regierer AC, Mariette X et al (2017) Spectrum of lymphomas across different drug treatment groups in rheumatoid arthritis: a European registries collaborative project. Ann Rheum Dis 76:2025-2030

15. Mielke J, Jilma B, Koenig F et al (2016) Clinical trials for authorized biosimilars in the European 
Union: a systematic review. Br J Clin Pharmacol 82:1444-1457

16. Richter A, Strangfeld A, Herzer P et al (2014) Sustainability of rituximab therapy in different treatment strategies: results of a 3-year followup of a German biologics register. Arthritis Care Res (Hoboken) 66:1627-1633

17. Strangfeld A, Eveslage M, Schneider M et al (2011) Treatment benefit or survival of the fittest: what drives the time-dependent decrease in serious infection rates under TNF inhibition and what does this imply for the individual patient? Ann Rheum Dis 70:1914-1920

18. Strangfeld A, Hierse F, Rau R et al (2010) Risk of incident or recurrent malignancies among patients with rheumatoid arthritis exposed to biologic therapy in the German biologics register RABBIT. Arthritis Res Ther 12:R5

19. Strangfeld A, Pattloch D, Herzer P et al (2013) Risk of cancer recurrence or new tumors in RA patients with prior malignancies treated with various biologic agents. 2013 ACR/ARHP Annual Meeting, October 25-30,2013 (Abstract Number:806)

\section{Bertelsmann-Stiftung: Mit weniger Kliniken bessere Versorgung}

Eine starke Verringerung der Klinikanzahl würde die Versorgungsqualität für Patienten verbessern und bestehende Engpässe bei Ärzten und Pflegepersonal mildern. Das zeigt eine neue Studie inklusive Modellberechnung im Auftrag der Bertelsmann Stiftung.

Eine Reduzierung der Klinikanzahl würde zu einer besseren medizinischen Versorgung der Patienten in Deutschland führen. In einer neuen Studie der Bertelsmann Stiftung weisen führende Krankenhausexperten darauf hin, dass viele Krankenhäuser in der Bundesrepublik Deutschland zu klein sind und oftmals nicht über die nötige Ausstattung und Erfahrung verfügen, um lebensbedrohliche Notfälle wie einen Herzinfarkt oder Schlaganfall angemessen zu behandeln. Viele Komplikationen und Todesfälle ließen sich durch eine Konzentration auf deutlich unter 600 statt heute knapp 1.400 Kliniken vermeiden. Ebenso gingen damit eine bessere Ausstattung, eine höhere Spezialisierung sowie eine bessere Betreuung durch Fachärzte und Pflegekräfte einher.

\section{Ziel ist mehr Patientensicherheit}

Das Berliner Institut für Gesundheits- und Sozialforschung (IGES) ist im Auftrag der Bertelsmann Stiftung der Frage nachgegangen, wie eine Versorgung durch Kliniken aussähe, die sich nicht in erster Linie an einer schnellen Erreichbarkeit, sondern an Qualitätskriterien orientiert. Dazu gehören beispielsweise eine gesicherte Notfallversorgung, eine Facharztbereitschaft rund um die Uhr, ausreichend Erfahrung und Routine des medizinischen Personals sowie eine angemessene technische Ausstattung.

\section{Modellregion Köln/Leverkusen}

Für die Studie haben laut BertelsmannStiftung führende deutsche Krankenhausexperten in einem ersten Schritt ein Zielbild für Deutschland entwickelt, das sich an diesen Qualitätskriterien orientiert. Im Anschluss berechnete das IGES in einer Simulation erstmals, wie sich eine verpflichtende Einhaltung dieser Vorgaben auf die Kliniklandschaft einer ganzen Region auswirken würde. Die Wahl fiel dabei auf den Großraum Köln/Leverkusen, der sowohl von städtischen als auch ländlichen Gebieten geprägt ist.
Wie die Simulation zeigt, könnte die Region mit 14 statt den aktuell 38 Akutkrankenhäusern eine bessere Versorgung bieten, ohne dass die Patienten im Durchschnitt viel längere Fahrzeiten in Kauf nehmen müssten. Die Bündelung von medizinischem Personal und Gerät würde zu einer höheren Versorgungsqualität in den verbleibenden Häusern beitragen, vor allem in der Notfallversorgung und bei planbaren Operationen. Nur diese Kliniken in der Region verfügen überhaupt über die technische Ausstattung, um Herzinfarktpatienten angemessen zu behandeln.

\section{Blick ins Ausland}

Tatsächlich zeige der Blick ins Ausland, dass es Potenzial für eine Verringerung der Klinikanzahl gebe. Deutschland weise im internationalen Vergleich im Durchschnitt mehr medizinisches Personal pro Einwohner auf als vergleichbare Länder, aber weniger pro Patient. Diese paradoxe Situation liege daran, dass in der Bundesrepublik viel mehr Patienten in Krankenhäusern versorgt würden als im Ausland. Wie Untersuchungen ergaben, müssten rund ein Viertel der heute in deutschen Kliniken behandelten Fälle nicht stationär versorgt werden, so die Bertelsmann-Stiftung.

Quelle: Bertelsmann-Stftung www.bertelsmann-stiftung.de 